\title{
Poster Abstract: On the Feasibility of High-power Radios In Sensor Networks
}

\author{
Cigdem Sengul, Mehedi Bakht, Albert F. Harris III, Tarek Abdelzaher and Robin Kravets \\ Email: \{sengul,mbakht2,aharris,zaher,rhk\}@cs.uiuc.edu \\ University of Illinois at Urbana-Champaign
}

\section{Introduction}

Wireless sensor nodes are constrained by limited energy resources. A significant portion of this limited energy is spent in communication, making energy efficiency of the radio a very important factor in increasing the overall lifetime of a sensor node. An ideal radio, in terms of energy efficiency (ignoring issues of transmission range and delay), should satisfy two requirements. First, it should minimize the energy used per bit when transmitting or receiving. Second, its idle time energy consumption should be minimal. Unfortunately, no such "ideal" radio exists. Current sensor radios (e.g., CC2420) meet the second requirement - the energy consumption in the inactive states is very low in comparison to IEEE 802.11 radios, which are comparatively energy-inefficient due to high switching-on cost and idle state energy consumption. On the other hand, IEEE 802.11 radios perform much better when it comes to energy consumption per bit. So the natural question to ask is whether there exists a way to combine the positive features of these two types of radios leading to increased energy conservation. We argue that this objective can indeed be achieved by adding a high-power IEEE 802.11 radio to the mote platform alongside the already existing sensor radio and managing them by an energyefficient scheme as proposed in this work.

Previous research has looked at adding low-power radio to high-power radio only platforms (e.g., PDA, Laptop). Those works showed that energy can be saved by using the additional low-power radio for device discovery and connection setup [1] or for waking up the high-power radio $[2,3]$. But in recent years, a different trend is emerging that advocates moving from the low-power radio only systems of current sensor platforms towards dual-radio systems comprising of both high and low power radios (e.g., Intel Stargate platform [4], LEAP (Low Power Energy Aware Processing) platform [5], Intel Mote 2 [6], [7]). These increased communication capabilities are causing a paradigm shift in sensor networks which were previously considered as low-data-rate, low-complexity networks. Resource-hungry applications, which range from ultra-low latency WSN routers [7] to sound or image collection, would greatly benefit from these new platforms. While creating such new opportunities, a multi-radio system also introduces new tradeoffs involving size of transmitted data and energy savings. Depending on the amount of data, it might be the case that switching-on cost and idle state energy consumption would make the use of high-power radios too expensive. The main contribution of this work is to propose a solution for this problem by identifying a feasible break-event point (in terms of data size) after which energy savings are possible by using the highpower radio.

\section{Feasibility of Multi-Radio Com- munication}

Since increasing network lifetime through energy conservation is the main concern in sensor networks, it is important to justify the cost of migration to a new multi-radio system from an energy perspective. To this end, we present in this section an energy-efficient scheme for managing radios in sensor nodes that are equipped with both a low-power/low-bandwidth radio (e.g., CC2400) and a high-power/high-bandwidth radio (e.g., IEEE $802.11 \mathrm{a} / \mathrm{b} / \mathrm{g}$ radio). The low-power sensor radio is used to maintain connectivity, while the IEEE 802.11 radio remains off unless it is switched on to send or receive. Nodes do not transmit data as soon as it is available. Instead, we propose accumulating data up to a certain amount. When there is sufficient data to justify the cost of sending by the high-power radio, the sender node switches that radio on and sends a wakeup message to the destination node (through the low-power radio) so that it switches on its high power radio for receiving.

To calculate the minimum amount up to which data should be accumulated by the nodes for energyefficient communication, which we will call the break-even point $s$, we first consider the case when only the sensor radio is used for communication. Given the transmission and reception costs $\left(P_{t x}^{S R}\right.$ and $\left.P_{r x}^{S R}\right)$ and the data rate $\left(R_{S R}\right)$ of the sensor radio, the 
energy cost of sending $s$ data is:

$$
E_{S R}(s, p s)=\left(P_{t x}^{S R}+P_{r x}^{S R}\right) \cdot \frac{s+\frac{s}{p s} \cdot h s}{R_{S R}},
$$

where $p s$ and $h s$ are packet size and header size respectively.

Next, we consider the case when the IEEE 802.11 radio is used for data transfer as described in our model. Assuming that the data rate for the interface is $R_{80211}$, the energy cost of sending $s$ data is:

$$
\begin{aligned}
& E_{802.11}\left(s, p s, R_{80211}\right)=E_{\text {wakeup }}+E_{S R}(\text { wakeup }) \\
& +E_{\text {idle }}+\left(P_{t x}^{802.11}+P_{r x}^{802.11}\right) \cdot \frac{s+\frac{s}{p s} \cdot h s}{R_{802.11}}
\end{aligned}
$$

where $E_{\text {wakeup }}$ is the energy spent in waking up the sender and receiver IEEE 802.11 radios (i.e., switching costs) and is simply $2 \cdot P_{\text {wakeup }} \cdot t_{\text {wakeup }}$. The energy cost of sending wake-up messages through the sensor radio is $E_{S R}($ wakeup $)$. $E_{\text {idle }}$ is the energy consumed by the two IEEE 802.11 radios when they remain in idle state for $t_{i d l e}$ amount of time in the expectation of data before being switched off. Hence, $E_{\text {idle }}=2 \cdot P_{\text {idle }} \cdot t_{\text {idle }}$.

To satisfy $E_{802.11}(s, R) \leq E_{S R}(s)$, the data should be at least:

$$
s \geq \frac{E_{\text {wakeup }}+E_{\text {idle }}+E_{S R}(\text { wakeup })}{\frac{P_{t x}^{S R}+P_{r x}^{S R}}{R_{S R}}-\frac{P_{t x}^{802.11}+P_{r x} 802.11}{R_{802.11}}}
$$

Based on (3), the break-even point, $s^{*}$, should satisfy $E_{802.11}\left(s^{*}\right)=E_{S R}\left(s^{*}\right)$.

So far, in comparing the energy cost of communication, we have assumed that the destination node can be reached by both the radios in a single hop. But typically, the transmission range of IEEE 802.11 radios is greater than that of sensor radios. Based on this discrepancy in transmission range, it is reasonable to assume that there will be cases when it takes multiple hops to reach a certain destination through only sensor radios, but the IEEE 802.11 radio can send to the same destination in a single hop for a given rate $R$. Denoting this hop progress $H P_{802.11}(R)$, energy consumption for each radio can be re-evaluated. While the energy consumption due to sensor radio communication changes to:

$$
E_{S R}^{\text {multihop }}(s)=H P_{802.11}(R) \cdot E_{S R}(s),
$$

the energy consumption of the IEEE 802.11 radio changes to:

$$
\begin{aligned}
& E_{802.11}^{\text {multihop }}\left(s, R_{80211}\right)=E_{802.11}\left(s, R_{80211}\right) \\
& +\left(H P_{802.11}(R)-1\right) \cdot E_{S R}(\text { wakeup }),
\end{aligned}
$$

which includes data transmission energy and the energy used for sending the wake-up message multiple hops.

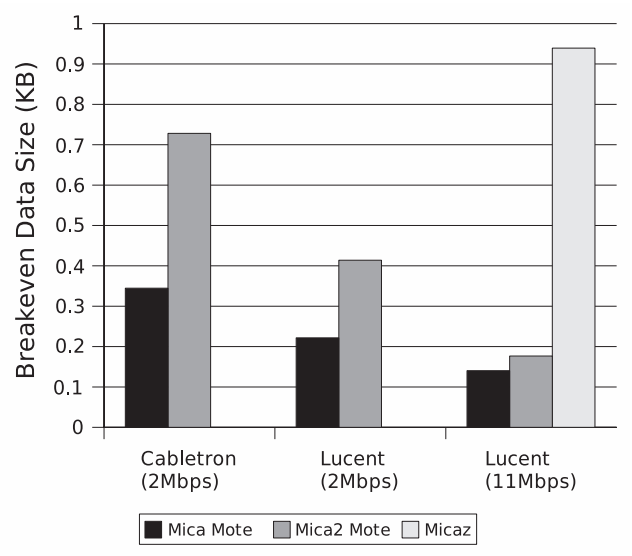

Figure 1: Break-even data size for different configurations. Idling energy is not included.

\section{Evaluation}

Having theoretically derived the condition for feasibility of energy savings through multi-radio systems, our next step is to explore the possibility of satisfying that condition with current radios. We start by evaluating how the break-even point changes for different combinations of IEEE 802.11 and sensor radios in the single hop case. Fig. 1 shows that the break-even point typically occurs at low data sizes (i.e., below $1 \mathrm{~KB}$ ). Not surprisingly, the data size to break even increases for more energy-efficient mote radios (i.e., Mica < Mica2 < Micaz) and decreases for more energy efficient IEEE 802.11 radios (i.e., Cabletron $>$ Lucent (2 Mbps) > Lucent (11 Mbps)). There are two cases where using a dual-radio approach is not feasible. For instance, when Micaz is used, both Cabletron and Lucent $(2 \mathrm{Mb} / \mathrm{s})$ do not provide any energy savings.

We next show the break-even point for the multihop case for the same IEEE 802.11 and sensor radio configurations. The transmission range of IEEE 802.11 radios is approximately $250 \mathrm{~m}$ and the sensor radios is $40 \mathrm{~m}$. To study the multi-hop case, we consider a linear topology of six nodes where the source and the destination are separated by $200 \mathrm{~m}$ and the intermediate nodes are evenly spaced. Hence, while the source can reach the destination in one hop with $\mathrm{Ca}$ bletron and Lucent (2 Mbps), communication through sensor radios requires 5 hops. Data rate and range for IEEE 802.11 radios are not independent parameters, but rather as the rate increases the range that can be supported decreases. Therefore, we assume Lucent (11 Mbps) has the same range as the sensor radio. As expected, the break-even point for Cabletron and Lucent $(2 \mathrm{Mbps})$ radios is lower for the multi-hop case (i.e., $0.15-0.75 \mathrm{~KB}$ ). Furthermore, Cabletron and Lucent (2 Mbps) configurations become feasible with 


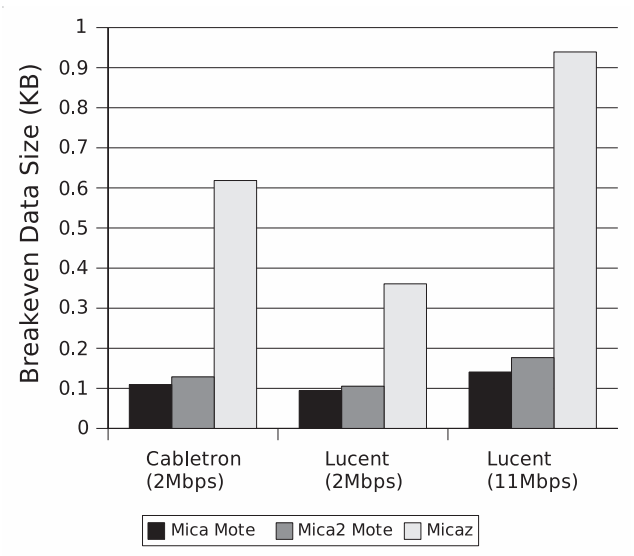

Figure 2: Breakeven data size for different configurations for multi-hop case. Idling energy is not included.

Micaz when forward progress of these radios are taken into account (Fig. 2). One interesting thing to note is that the break-even point for Lucent (11 Mbps) is the same in the multi-hop case as the single hop case. This is because both the energy spent in waking up and energy spent in communication increase with hop count, hence, cancel each other.

For both single-hop and multi-hop case, the breakeven point occurs typically at $1 \mathrm{~KB}$, which corresponds to approximately one packet for 802.11 radios. Further energy savings can be gained if it is possible to go over the breakeven point and send the collected data in larger bulks. But as the burst size increases, diminishing returns on energy savings are expected. Fig. 3 shows the energy savings obtained from sending $n$ packets in one shot in comparison to waking up $n$ times and sending 1 packet at each awake period. Energy savings increase fast up to 10 packets (i.e. $10 \mathrm{~KB}$ ) and then continue increasing with a slower rate, which suggests that a burst size of around $10 \mathrm{~KB}$ would be a good operating point.

\section{Summary and Future Work}

Additon of high-power radios creates new challenges and possibilities for sensor networks. In this work, we studied the issues from a local perspective and showed that it is feasible to save energy through the dual-radio system if we can buffer only few KBs of data and then send it through the high-power radio. In future, we plan to evaluate the dual-radio system from a network perspective and investigate the impact of adding highpower radios on congestion and contention. We also plan to compare the performance of our multi-radio communication model with the existing single-radio system by implementing our proposed scheme on new dual-radio sensor platforms (e.g., LEAP [5]).

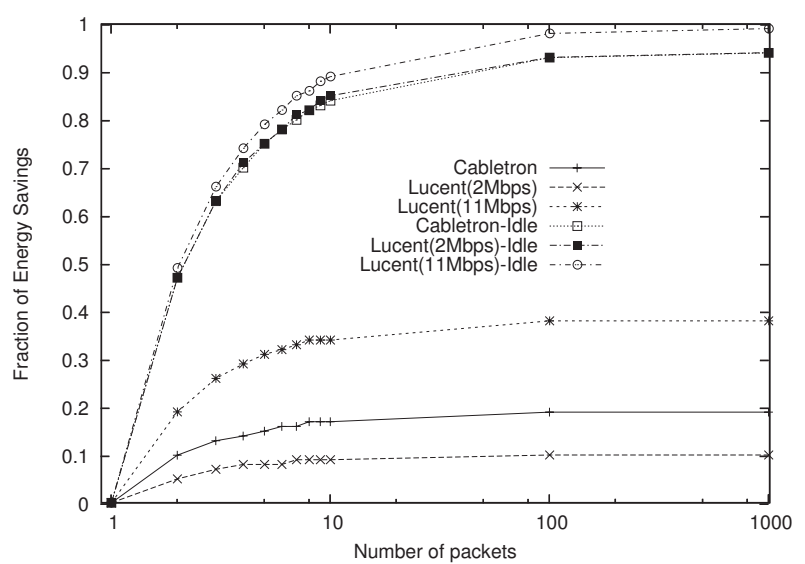

Figure 3: Fraction of energy savings as the number of consecutively sent packets increases. Each packet is $1 \mathrm{~KB}$. When idling energy is taken into account, idle time threshold is set to $100 \mathrm{~ms}$.

\section{References}

[1] T. Pering, V. Raghunathan, and R. Want, "Exploiting radio hierarchies for power-efficient wireless device discovery and connection setup," in 18th International Conference on VLSI Design, January 2005.

[2] E. Shih, P. Bahl, and M. J. Sinclair, "Wake on wireless: An event driven energy saving strategy for battery operated devices," in Mobile Computing and Networking (MobiCom), September 2002.

[3] J. Redi, S. Kolek, K. Manning, C. Partridge, R. Rosales-Hain, R. Ramanathan, and I. Castineyra, "JAVeLEN- An ultra-low energy ad hoc wireless network," BBN, Tech. Rep., 2006.

[4] "Intel stargate platform," http://platformx. sourceforge.net/index.html.

[5] D. McIntire, K. Ho, B. Yip, A. Singh, W. Wu, and W. J. Kaiser, "The low power energy aware processing (LEAP) embedded sensor system," in Information Processing in Sensor Networks (IPSN), April 2006.

[6] "Intel mote 2 overview," http://www.intel. com/research/downloads/imote_overview.pdf, September 2005.

[7] M. Kohvakka, "Atific helicopter high performance multi-radio WSN platform," White paper http://atific.fi/files/helicopter/ AtificHelicopter_WhitePaper.pdf, May 2006. 